\title{
A Novel Hardware Approach to Integrating Active and Passive Rehabilitation in a Single Exoskeleton
}

\author{
Soumya K Manna ${ }^{1}$, Venketesh N. Dubey ${ }^{2}$ \\ Faculty of Science \& Technology \\ Bournemouth University \\ Bournemouth, United Kingdom \\ 1'smanna@bournemouth.ac.uk, ${ }^{2}$ vdubey@bournemouth.ac.uk
}

\begin{abstract}
The proposed exoskeleton is based on the elbow joint where patients can have active and passive rehabilitation in a single structure without changing its configuration. The structural formation of the exoskeleton has been designed in such a way that it offers two working regions namely; actuator based active rehabilitation in the first phase and passive rehabilitation in the second phase. The solution for integrating these two phases has been implemented using an innovative passive locking mechanism which uses a spring-based system for transformation. The stiffness of the spring is utilized to switch between active and passive rehabilitation regions. Besides this there are some other advantages this exoskeleton offers such as reduction of the actuation torque as well as ease of control. The paper is divided into three parts: the first part describes the existing designs, the second part gives an overview of the developed mechanism with structural description and the last part provides the solution with technical specification.
\end{abstract}

Keywords - exoskeleton, rehabilitation, locking mechanism, portability

\section{INTRODUCTION}

At present, there are over 1.2 million stroke survivors in the UK as per the Stroke Association [1]. In terms of intensive and cost-effective therapy, exoskeleton based rehabilitation is considered to be superior to manual therapy [2]. However, the way of recovery of stroke patients depends on the process of rehabilitation which includes all the orthopedic lessons related to active and passive rehabilitation [3].

Active actuator based rehabilitation is applicable to acute stroke patients. In the acute phase, there is no joint movement due to lack of coordination between muscle and brain. Therefore, human joints need to be put in action by applying external force using exoskeleton. At the early stage, patients are rehabilitated with some predefined orthopedic lessons at different frequencies. This type of rehabilitation can be called either active or passive from two different perspectives. It may be called as passive rehabilitation from the patient's point of view as they don't have active participation and all motions are controlled by the external actuator used in the exoskeleton. Sometimes this is also referred to as active rehabilitation because exoskeleton is providing all the motions. To avoid the confusion, the second perspective (exoskeleton's point of view) is used in this paper. Patients usually regain a small amount of muscle strength to initiate their joint movements after active rehabilitation. However, they face a great deal of difficulty in balancing their arms. Hence, a support mechanism would be helpful to assist those patients to continue their movements for different exercises as well as daily activities. The supportive force would encourage those patients to engage in more effort during exercise and their neuro-motor function will be improved gradually. Exercise in this phase is called passive because patients can initiate their joint movement and the exoskeleton is just performing the supportive role.

The exercises involved in different rehabilitation stages recover the muscle strength to get them back to the normal life. Therefore, exoskeleton needs to provide all kinds of exercises related to active and passive rehabilitation to make a better recovery process. It is nevertheless an easy task to achieve active and passive rehabilitation in a single mechanism because exercises associated with both types of rehabilitation are contradictory in nature. A system providing active rehabilitation should require firm contact with human arm during training; while passive rehabilitation based system requires flexible or loose contact to carry out the exercises according to the triggering pattern of the patient.

Active and passive rehabilitation can be incorporated in an arm exoskeleton either by hardware or software approaches. The software-based solution is comparatively easy from a construction point of view because the actuator is placed at the joint and the desired joint torque can be maintained using feedback sensor and soft computing techniques [4]. Usually electric motor (brushed and brushless) is the most popular option [5] as an actuator because of its linear and easy to control technique. This technique involves adaptive control system where motor torque will be varied depending on the patient's effort taken from different biosensors attached to the human body. However, there are some limitations regarding stability and feedback constraints. It is quite difficult to exert exact muscle signal from post-stroke patients using EMG [6]. EEG based brain-computer interface may be superior in terms of extracting the signal but it is difficult to recognize the type of action by analyzing the EEG signal [7]. Due to the dependency on biosignals those devices are inoperable without sensors. The aim of post-stroke rehabilitation is to increase the patient's effort through exercises, therefore a portion of the required joint torque needs to be provided by exoskeleton during rehabilitation and the amount of torque should be decreased with time to increase the patient's involvement. Adaptive control system relies on assistance as needed strategy 
[8]. A recent study of different adaptive control system proved that there is an adverse effect of this technique; it has been found that the exoskeleton takes full control of the joint motion by providing the entire required torque through assistance, therefore makes the patient passive which reduces the patient's involvement in the movement and so is the recovery rate [9]. This technique could be useful as an assistive device but not for the rehabilitation purpose. In software solutions, delays exist thus this may exhibit a discordant behavior on sudden impact force. Also neurological patients may suffer from painful and involuntary muscular contraction which may lead to a joint stiffness with undesirable torque [10]. A sophisticated control algorithm used for controlling the variable joint torque and active range of motion all the time may result in constant draining of energy, thus may increase the size of the energy source and reduces the portability of the system. Also the joint based actuation will require maximum torque for moving a segment of the arm. Therefore, the size, weight, power consumption and cost of the motor are increased based on the torque level. In this technique the human joint is always under motor control which may not be safe. If the motor moves beyond the anatomical limit of the human joint due to malfunction or delays, accident might happen. Therefore, it may be a better idea to go for a hardware solution to overcome these drawbacks. Rehabilitation can also be accomplished using different hardware-based solutions. This approach can reduce the complexity in control system by allowing active and passive components in the structure. Generally separate exoskeletons are used to achieve active or passive rehabilitation. Different actuation such as electric motor, hydraulic [11] and pneumatic [12] drives are used for active rehabilitation whereas passive rehabilitation uses elastic elements such as spring [13] or rubber band [14]. Spring-based mechanism can create energy-less system because no active actuator is involved. However, there are no hardware based arm exoskeleton which integrates both types of rehabilitation and preserves its portability and efficiency. A few hardwarebased exoskeletons which consider both types of rehabilitation are based on hand functions such as iHandRehab [15]. The existing systems use more actuators to accommodate all types of exercises which may create problem for portability. Such systems use the electromagnetic switches for shifting between rehabilitation modes [16]; it may drain some energy and create unwanted noise during switching.

\section{MECHANICAL DESIGN}

To overcome all these problems, a locking mechanism is introduced in this paper which will help the exoskeleton to serve both active and passive rehabilitation in a single structure. Two rehabilitation regimes are interconnected using a spring-actuated lock and will appear one after another automatically; therefore, no need to change the structure or press any switch to achieve it. The lock will be closed in active rehabilitation mode to provide controlled joint movement using electric motor whereas it will be open in passive rehabilitation mode to provide flexible support using springs. Instead of using an electromagnetic switch, spring stiffness is used for the locking and unlocking operation. Depending on the recovery, the mode of rehabilitation can be changed by changing the mode of operation. The developed technique reduces the dependency on the biosensor and the mode of rehabilitation can also be altered manually. A single motor is used in this exoskeleton to achieve all these features. The required motor torque for joint rotation has been considerably reduced by using this exoskeleton. The part of the exoskeleton used for passive rehabilitation can also work under no power condition. The exoskeleton is structurally safe for controlling the range of elbow rotation to the anatomical limit in active rehabilitation.

\section{A. Construction}

The exoskeleton has been designed based on elbow joint because it is one of the simplest human arm joints having one degree of freedom. The working principle of the proposed exoskeleton has been configured in view of the standard rehabilitation procedure which consists of a few sequential stages providing a specific type of rehabilitation. Both schematic and 3D model of the proposed exoskeleton are shown in Fig. 1 and 2 respectively in locked and unlocked conditions. In order to reduce the size and weight of the actuator, the proposed exoskeleton has been developed based on a lead-screw driven joint followed by a slider-crank mechanism as shown in Fig. 1. The lead screw and crank are not directly coupled to each other. There are two guiding rods working as slider along the lead-screw, one of which acts as a nut and another is concentric to the lead-screw, not connected to it. However, the nut slider translates in both directions following the guiding path of screw thread as per the rotation of the motor. The other slider can only slide on the screw concentrically. Those sliders are also supported by another solid rod which helps them to maintain the linear sliding motion during movement. In both Fig. 1 and 2, it is shown that the concentric slider is connected to the crank using a connecting link on the right-hand side whereas an extension spring $\left(\mathrm{S}_{1}\right)$, used for passive rehabilitation, is connected to the same concentric slider on the left-hand side.

As per the design, the movement of the nut slider is controlled by the motor. Attachment of both sliders constructs the situation of active rehabilitation where crank rotation is fully controlled by the motor (Fig. 1, Locked condition). A fixed number of clockwise and anticlockwise rotation of the motor allows the active rehabilitation of the elbow joint. A slight increment in the motor rotation beyond the range releases the lock and shifts the working region into passive one. There won't be any effect of motor rotation on crank movement if both sliders are not connected together in the pulling direction. This situation will switch to passive rehabilitation where patients will generate their movements and the required joint torque is supported by $\mathrm{S}_{1}$ (Fig. 1, Unlocked condition). Two compression springs $\left(\mathrm{S}_{2}\right.$ and $\left.\mathrm{S}_{3}\right)$ of different stiffness help to switch between active to passive rehabilitation regime. $\mathrm{S}_{2}$ is attached to the baseplate and provides the opposite force for opening of the lock. $\mathrm{S}_{3}$ is used by the lock to latch both sliders during active rehabilitation using two clawtype jaws (Fig. 4). Spring force of the lock generated by $\mathrm{S}_{3}$ maintains its two jaws parallel during active rehabilitation. The stiffness of $\mathrm{S}_{3}$ should be as low as possible so that a small opposite force is higher enough to open its two jaws. The stiffness of $\mathrm{S}_{2}$ should be higher than that of $\mathrm{S}_{3}$ so that a small displacement in $\mathrm{S}_{2}$ can cause a large displacement in $\mathrm{S}_{3}$, strong enough to open the lock. The mechanism of the exoskeleton 
has been designed in such a way that if the whole region of active rehabilitation is covered, the elbow joint will rotate the full range of motion $\left(130^{\circ}\right)$, therefore no hyperextension of the joint. If the position of the nut slider goes beyond the active rehabilitation, it will automatically open up the lock and change the mode of rehabilitation where elbow joint motion would not be controlled by the motor.

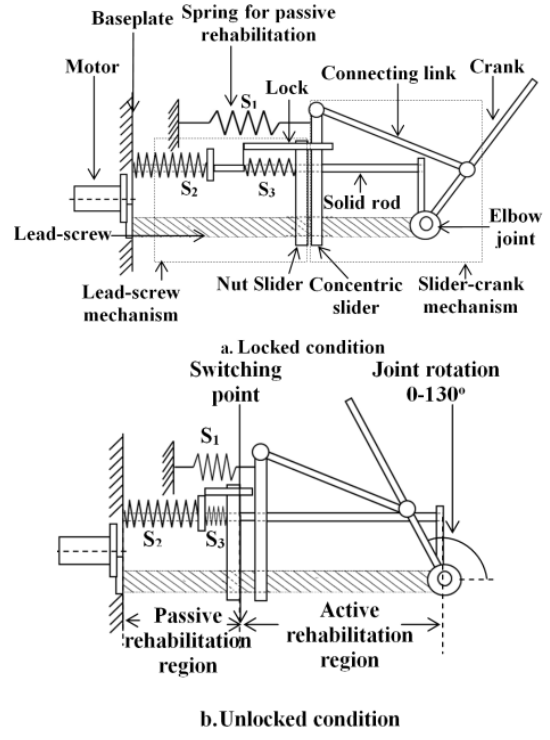

Fig. 1. Schematic diagram of exoskeleton
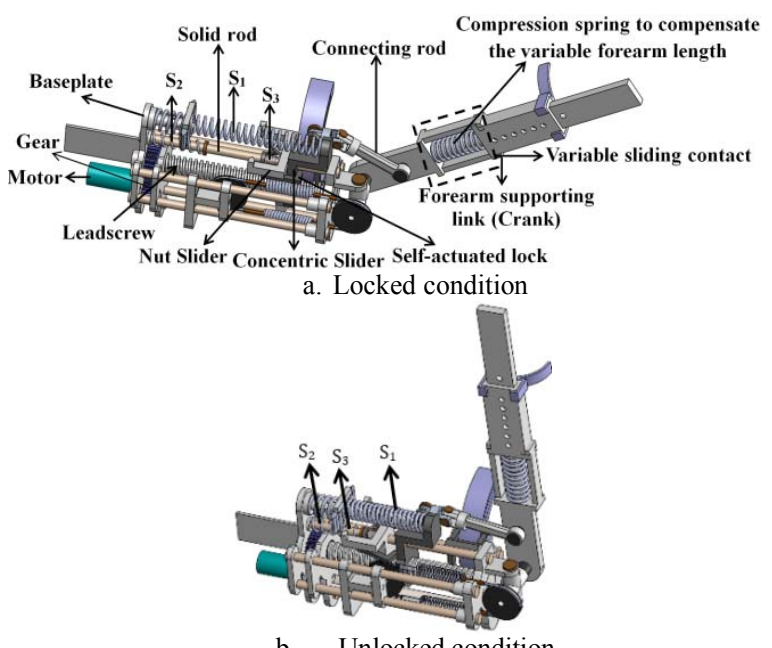

Fig. 2. 3D model of exoskeleton

The forearm has a prismatic joint with a compression spring which can compensate for any misalignments between exoskeleton and the user (Fig. 3).

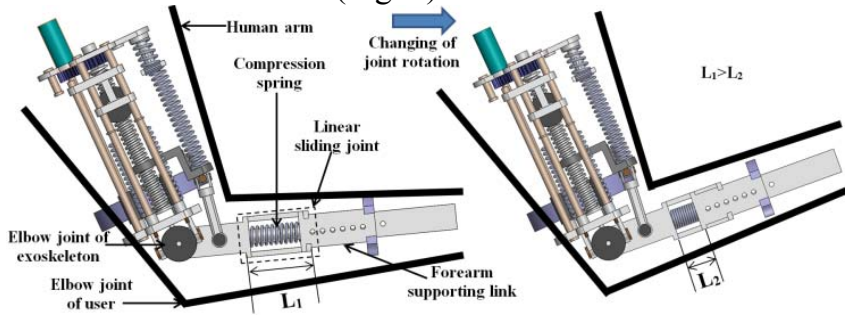

Fig. 3. Misalignment adjustment on the forearm

\section{B. Operation}

The elbow joint can rotate up to $130^{\circ}$ during active rehabilitation. A closer view of the locking mechanism shows that those two jaws are connected between nut slider and $S_{3}$ in a form of four-bar mechanism (Fig. 4, Locked condition). Due to the backward movement of the nut slider beyond the active rehabilitation regime, the slider assembly with the lock pushes $\mathrm{S}_{2}$. Since the stiffness of $\mathrm{S}_{2}$ is higher compared to $\mathrm{S}_{3}$, the force exerted by $\mathrm{S}_{2}$ is greater than the later. As a result, $\mathrm{S}_{3}$ would be compressed by the resultant force which drives its two jaws to rotate about a fixed point, see unlocked condition in Fig. 4. This action will free the concentric slider from the lock and thereafter joint movement will be controlled by the spring $\left(\mathrm{S}_{1}\right)$. If the nut slider moves backward towards the baseplate, the lock will remain open. Due to the mechanical restriction from the nut slider, $\mathrm{S}_{3}$ can't be compressed beyond a certain point; however, $\mathrm{S}_{2}$ will be compressed to compensate the variation of the distance between the nut slider and the baseplate. The forward movement of the nut slider beyond the switching point will put the mechanism into the active rehabilitation mode; therefore, $\mathrm{S}_{3}$ will move again to its normal state to hold both sliders.

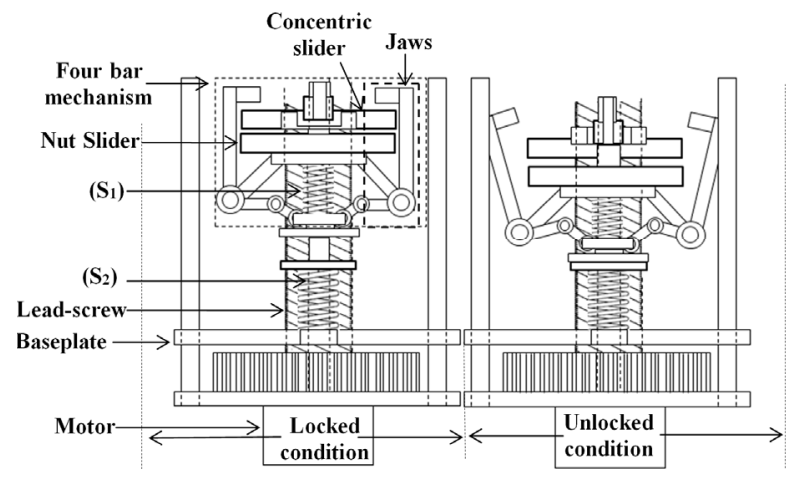

Fig. 4. Schematic diagram of the locking mechanism

\section{Prototype of the exoskeleton}

Based on the above design considerations a prototype is developed to establish the working principle of the exoskeleton. All customized components are made using ABS (Acrylonitrile butadiene styrene). The overall weight of the exoskeleton is $1.8 \mathrm{~kg}$. A passive structure consisting of buckles is attached to the shoulder to support the load of the exoskeleton. The prototype is shown in Fig. 5, the size and weight could be minimized in future iterations.

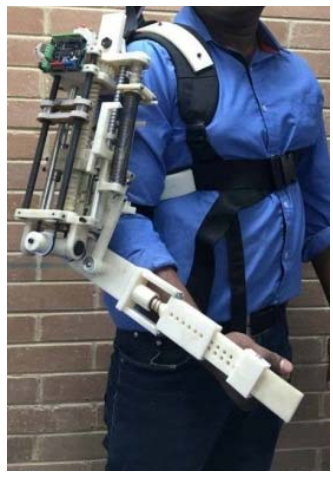

Fig. 5. The prototype of the elbow exoskeleton 


\section{STATIC ANALYSIS}

\section{A. Active rehabilitation mode}

As discussed in the design part, the elbow joint of the exoskeleton is actuated using a slider crank mechanism (Fig. 6) to reduce the required torque for rotating the joint. Fig. 7 shows that the required maximum motor torque is significantly reduced using the exoskeleton for different weight of the forearm.

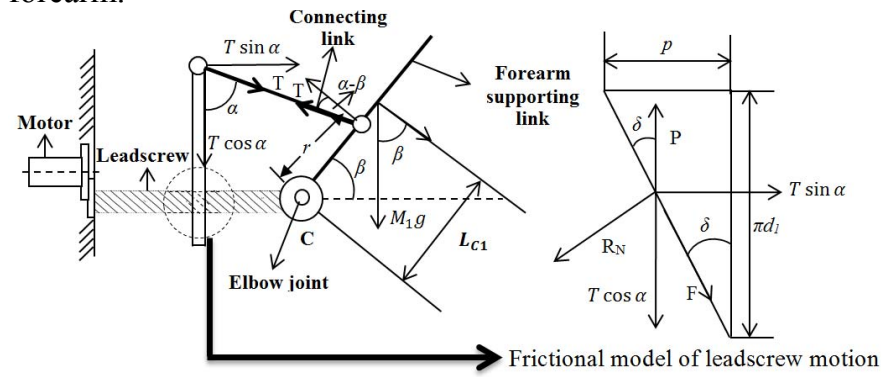

Fig. 6. Mechanism in active rehabilitation

If the motor is connected to the elbow joint, the required motor torque $\left(\tau_{j}\right)$ for rotating the elbow is given by

$$
\tau_{j}=M_{1} g * L_{C 1} \cos \beta
$$

The actuation force in the proposed exoskeleton is provided using the pulling force. The required force $(T)$ for lifting up the weight of the forearm is

$$
T=\frac{M_{1} g * L_{C 1} \cos \beta}{r \cos (\alpha-\beta)}
$$

Where, $M_{1} g=$ Weight of the forearm and the supporting link $L_{C l}=$ Length of center of mass of the forearm and the supporting link

$\beta=$ Joint angle

$\alpha=$ Angle between the connecting rod and slider

$r=$ Length of the crank

The frictional model of the leadscrew provides

$$
\tan \delta=\frac{p}{\pi d_{1}}
$$

Where, $p=$ Pitch of the leadscrew,

$d_{l}=$ Diameter of the leadscrew

$\delta=$ Lead angle of leadscrew

In active rehabilitation, the motor provides the torque to overcome the frictional force in the leadscrew. Effort $(P)$ is applied at the circumference of the screw to lift the load. Writing the force equilibrium in the frictional model (Fig. 6),

$$
\text { We have, } P \cos \delta=W \cos \delta+W_{1} \sin \delta+F
$$

Where, $T \cos \alpha=W$ and $T \sin \alpha=W_{l}$

and Frictional force $(F)$ during motion is

$$
F=\mu R_{N}=\mu\left(W \sin \delta-W_{1} \cos \delta-P \sin \delta\right)
$$

$\mu=$ Coefficient of friction

After substituting the value of $F$ in (4), we get

$$
P=W+W_{1} \frac{(\sin \delta-\mu \cos \delta)}{(\cos \delta+\mu \sin \delta)}
$$

Substituting $\mu=\tan \varphi(\varphi$ being the friction angle) in (6),

$$
P=W+W_{1} \tan (\delta-\varphi)
$$

After substituting the value of $W$ and $W_{l}$, we have

$$
P=T(\cos \alpha+\sin \alpha \tan (\delta-\varphi))
$$

Torque $\left(\tau_{e}\right)$ required for overcoming the frictional force of the leadscrew is

$$
\tau=P * \frac{d_{1}}{2}=\frac{T(\cos \alpha+\sin \alpha \tan (\delta-\varphi)) * d_{1}}{2}
$$

Putting the value of $T$ from (2), the final equation of required motor torque $\left(\tau_{e}\right)$ for the exoskeleton is

$$
\tau_{e}=\frac{M_{1} g * L_{C 1} \cos \beta(\cos \alpha+\sin \alpha \tan (\delta-\varphi)) * d_{1}}{2 r \cos (\alpha-\beta)}
$$

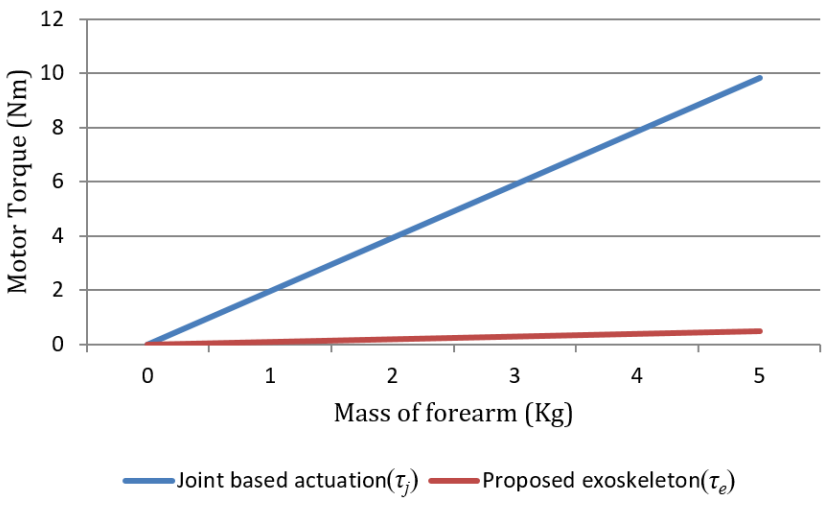

Fig. 7. Comparative torque between the exoskeleton and joint based actuator

\section{B. Passive rehabilitation mode}

As per Fig. 8, $\mathrm{S}_{1}$ is extended to share a portion of the required joint torque during passive rehabilitation. The stiffness of $S_{1}$ can be determined based on the weight of the forearm of the patient as shown in Fig. 9. However, the value of stiffness of $S_{1}$ should be high enough to support different forearm weight range.

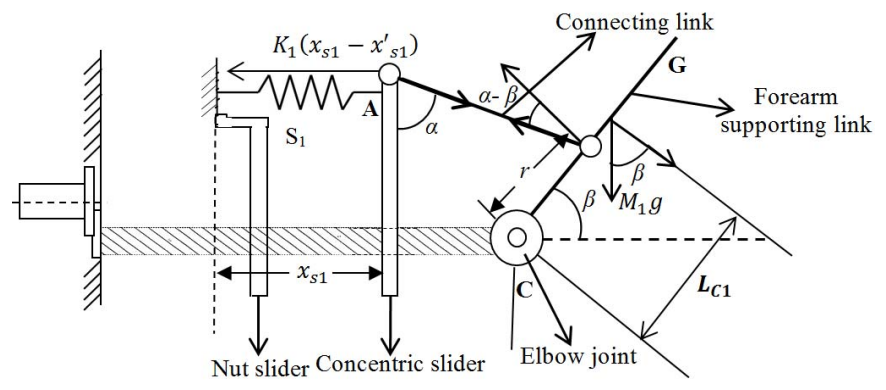

Fig. 8. Mechanism in passive rehabilitation

Tension along the connecting link is the same as it is during active rehabilitation. Therefore, the value of $T$ is taken from Eq. (2). The only difference is that $S_{1}$ is taking care of the load instead of the motor,

By equilibrating the forces at point $\mathrm{A}$, we have

$$
T \sin \alpha=K_{1}\left(x_{s 1}-x_{s 1}^{\prime}\right)
$$

Where, $x_{s l}=$ Displacement of $\mathrm{S}_{1}, x^{\prime}{ }_{s I}=$ Free length of $\mathrm{S}_{1}$

Putting the value of $\mathrm{T}$, taken from (2) in (11), the required stiffness of $\mathrm{S}_{1}$ is given by

$$
K_{1}=\frac{M_{1} g * L_{C 1} \cos \beta * \sin \alpha}{r \cos (\alpha-\beta) *\left(x_{s 1}-x_{s 1}\right)}
$$




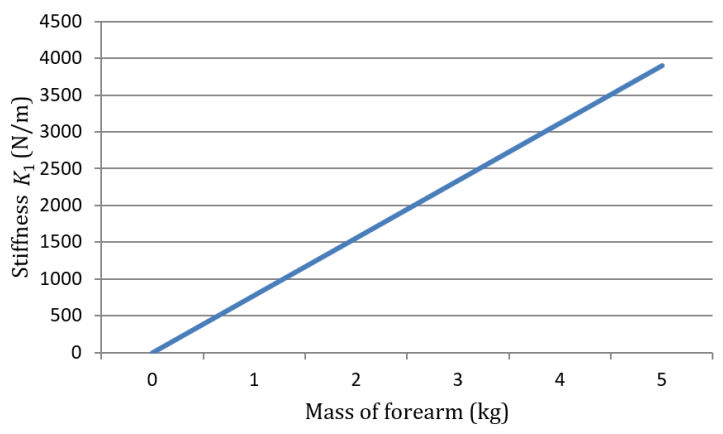

Fig. 9. Stiffness requirement of $S_{1}$ for different forearm mass

\section{Switching between two rehabilitation modes}

Fig. 10 shows the force balancing diagram of the mechanism during unlocked condition. The ratio of stiffness between both springs $\left(\mathrm{S}_{2}\right.$ and $\left.\mathrm{S}_{3}\right)$ can be determined depending on the structural parameters of the locking mechanism.

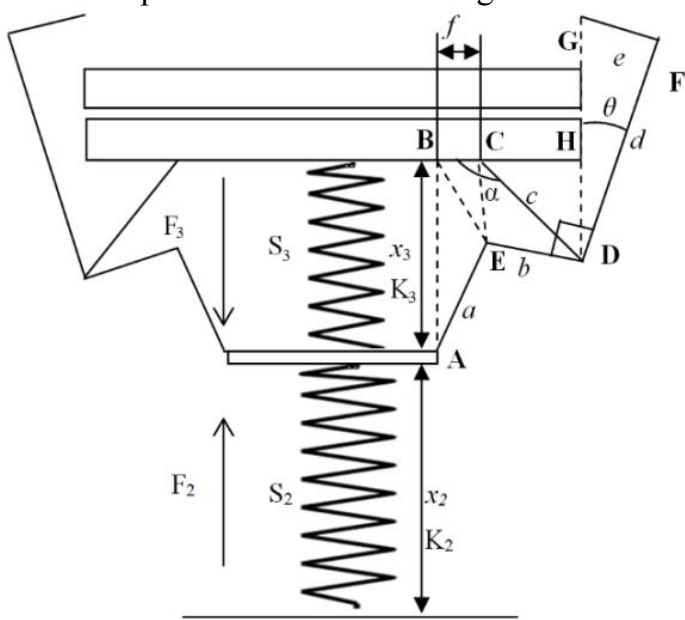

Fig. 10. Force balancing diagram during the unlocked condition

The mechanism can only function if it satisfies the following condition, $\mathrm{K}_{2} \gg \mathrm{K}_{3}$

Which means, $x_{2} \ll x_{3}$

Where, $\mathrm{K}_{2}, \mathrm{~K}_{3}=$ Stiffness of $\mathrm{S}_{2}$ and $\mathrm{S}_{3}$ respectively $x_{2}, x_{3}=$ Displacement covered by $\mathrm{S}_{2}$ and $\mathrm{S}_{3}$ respectively

After opening of the lock, both springs will be in equilibrium condition which means force exerted by $S_{2}$ and $S_{3}$ will be same at that position.

Therefore,

$$
\begin{gathered}
\mathrm{F}_{2}=\mathrm{F}_{3} \\
\mathrm{~K}_{2} x_{2}=\mathrm{K}_{3} x_{3} \\
\mathrm{~K}_{2}=\frac{\mathrm{K}_{3} x_{3}}{x_{2}}
\end{gathered}
$$

For the right-sided jaw shown as EDFG, the upper-end position of the jaw $\mathrm{G}$ should be outside of the region covered by those sliders (shown as the dotted line GD). It is clear that those two jaws need to rotate a minimum angle ' $\theta$ ' about the point $\mathrm{D}$ to unlock the concentric slider from locking range. The required angle $\theta$ for unlocking concentric slider from the jaw is

$$
\theta=\tan ^{-1} \frac{e}{d}
$$

Where, $a, b, c, d, e, f$ - length of the solid links used in the locking mechanism are constant and $\angle \mathrm{BCD}=\alpha ; \angle \mathrm{EDF}=90^{\circ}$.
As the values of $e$ and $d$ are constant, the value of $\theta$ is defined for unlocking condition. To achieve the angle, $\mathrm{S}_{3}$ needs to move a particular distance which can be derived by the geometrical considerations using all other construction parameters.

In Fig. 10, it shows that

and

$$
\angle \mathrm{CDH}=\left(\alpha-90^{\circ}\right)
$$

$$
\angle \mathrm{CDE}=\left(180^{\circ}-(\alpha+\theta)\right)=\left(180^{\circ}-\left(\alpha+\tan ^{-1} \frac{e}{d}\right)\right)
$$

From $\triangle \mathrm{CDE}$, it can be derived that,

$$
\cos (\angle \mathrm{CDE})=\frac{b^{2}+c^{2}-\mathrm{CE}^{2}}{2 b c}
$$

Substituting the value of $\angle \mathrm{CDE}$ from (16), the value of $\mathrm{CE}$ can be obtained as shown in (17)

$$
\begin{aligned}
& \cos \left(180^{\circ}-\left(\alpha+\tan ^{-1} \frac{e}{d}\right)\right)=\frac{b^{2}+c^{2}-\mathrm{CE}^{2}}{2 b c} \\
& \mathrm{CE}^{2}=b^{2}+c^{2}+2 b c * \cos \left(\alpha+\tan ^{-1} \frac{e}{d}\right)
\end{aligned}
$$

From $\triangle \mathrm{CDE}$, it can also be shown that,

$$
\cos \angle \mathrm{DCE}=\frac{c^{2}+\mathrm{CE}^{2}-b^{2}}{2 c * \mathrm{CE}}
$$

Using the value of CE taken from (17), we get the value of $\angle \mathrm{DCE}$

$$
\angle \mathrm{DCE}=\cos ^{-1}\left(\frac{c+b * \cos \left(\alpha+\tan ^{-1} \frac{e}{d}\right)}{\sqrt{b^{2}+c^{2}+2 b c * \cos \left(\alpha+\tan ^{-1} \frac{e}{d}\right)}}\right)
$$

$\angle \mathrm{BCE}$ can be written as $(\alpha-\angle \mathrm{DCE})$

Substituting the value of $\angle D C E$ taken from (18) in the above equation,

$$
\angle \mathrm{BCE}=\alpha-\cos ^{-1}\left(\frac{c+b * \cos \left(\alpha+\tan ^{-1} \frac{e}{d}\right)}{\sqrt{b^{2}+c^{2}+2 b c * \cos \left(\alpha+\tan ^{-1} \frac{e}{d}\right)}}\right)
$$

From $\triangle \mathrm{BCE}$, it can be seen that,

$$
\mathrm{BE}^{2}=f^{2}+\mathrm{CE}^{2}-2 f * \mathrm{CE} * \cos \angle \mathrm{BCE}
$$

Substituting the value of CE (from (17)) and $\angle B C E$ (from (19)) in (20), we can get

$$
\mathrm{BE}^{2}=f^{2}+A_{1}-2 f \sqrt{A_{1}} * \cos \left(\delta-\cos ^{-1}\left(\frac{B_{1}}{\sqrt{A_{1}}}\right)\right)
$$

Where, $A_{1}=b^{2}+c^{2}+2 b c * \cos \left(\delta+\tan ^{-1} \frac{e}{d}\right)$

$\left.B_{1}=c+b * \cos \left(\delta+\tan ^{-1} \frac{e}{d}\right)\right]$

From $\triangle \mathrm{BCE}$, we can get the value of $\angle \mathrm{CBE}$.

$$
\cos \angle \mathrm{CBE}=\frac{f^{2}+\mathrm{BE}^{2}-\mathrm{CE}^{2}}{2 f * \mathrm{BE}}
$$

After substituting the value of $\mathrm{CE}$ and $\mathrm{BE}$ taken from (17) and (21) respectively, we can express $\angle \mathrm{CBE}$ as

$$
\angle \mathrm{CBE}=\cos ^{-1}\left(\frac{f-\sqrt{A_{1}} * \cos \left(\delta-\cos ^{-1}\left(\frac{B_{1}}{\sqrt{A_{1}}}\right)\right)}{f^{2}+A_{1}-2 f \sqrt{A_{1}} * \cos \left(\delta-\cos ^{-1}\left(\frac{B_{1}}{\sqrt{A_{1}}}\right)\right)}\right)
$$

During unlocking condition, the displacement made by $\mathrm{S}_{3}$ create $\triangle \mathrm{ABE}$ where $\angle \mathrm{ABE}$ can be written as $\left(90^{\circ}-\angle \mathrm{CBE}\right)$ 


$$
\cos \left(90^{\circ}-\angle \mathrm{CBE}\right)=\frac{x_{3}{ }^{2}+\mathrm{BE}^{2}-a^{2}}{2 * x_{3} * \mathrm{BE}}
$$

After putting all the structural parameters $(\angle \mathrm{CBE}$ and $\mathrm{BE})$ of the design in (23), we have, $x_{3}=1.5 \mathrm{~cm}$ (approximately).

Where, $a=1.25 \mathrm{~cm}, b=1.375 \mathrm{~cm}, c=1.5 \mathrm{~cm}, d=4.6 \mathrm{~cm}, e=1.25$ $\mathrm{cm}, f=0.75 \mathrm{~cm}$ and $\alpha=135.61^{\circ}$

Primary length of $x_{3}=2 \mathrm{~cm}$ (in unlocked condition)

Therefore, total displacement $\Delta x_{3}=(2-1.5)=0.5 \mathrm{~cm}$

It stated before in the operation section that $\mathrm{K}_{2}$ needs to be higher than $\mathrm{K}_{3}$ so that the desired value of $x_{3}$ (displacement by $\mathrm{S}_{3}$ ) for the unlocking condition can be achieved for a very small displacement of $\mathrm{S}_{2}\left(x_{2}\right)$. The switching region should be as small as possible to make a clear boundary between two rehabilitation regions. Therefore, we assume that $x_{3}$ moves 0.5 $\mathrm{cm}$ for the displacement of $x_{2}=0.1 \mathrm{~cm}$ for proper functioning,

Putting the value of $x_{3}$ and $x_{2}$ in (13), we get

$$
\mathrm{K}_{2}=5 \mathrm{~K}_{3}
$$

After putting the dimension of all components and value of all other parameters, the value of $\mathrm{K}_{3}=80 \mathrm{~N} / \mathrm{m}$

Therefore, the value of $K_{2}$ should be at least $400 \mathrm{~N} / \mathrm{m}$ to satisfy the unlocking condition.

During active rehabilitation, $\mathrm{S}_{3}$ does not experience any force. At the start of passive rehabilitation, it will get an opposite force produced by $S_{2}$. Due to the mechanical restriction from the nut slider, $x_{3}$ won't change anymore after unlocking which results in a constant spring energy possessed by $\mathrm{S}_{3}$ throughout the rest of the motion.

Maximum force experience by $\mathrm{S}_{3}=80 \times 0.0050=0.4 \mathrm{~N}$ (as the maximum value of $x_{3}=0.5 \mathrm{~cm}=0.0050 \mathrm{~m}$ )

However $\mathrm{S}_{2}$ will be compressed again due to further movement of nut slider and $x_{2}$ will change as well. Fig. 11 shows the force diagram of both springs $\left(S_{2}\right.$ and $\left.S_{3}\right)$ with respect to the position of the nut slider.

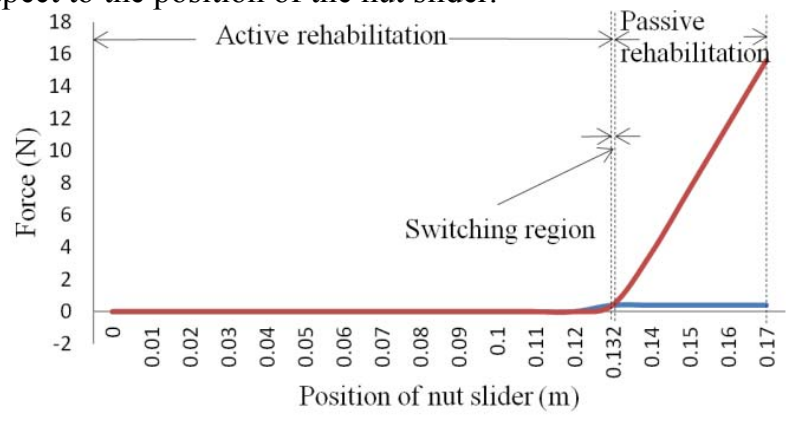

-Force experienced by $\mathrm{S}_{3} \quad$ Force experienced by $\mathrm{S}_{2}$

Fig. 11. Force experienced by both springs $\left(\mathrm{S}_{2}\right.$ and $\left.\mathrm{S}_{3}\right)$

\section{CONCLUSION}

The paper presented a mechanism that could provide both active and passive rehabilitation depending on the patient requirements. Though the hardware approach requires much more complex mechanism, still it may be useful for humanmachine interactions due to easy control and safety considerations. The reasons of dividing the whole working region into two types of rehabilitation offer flexibility to choose between two conditions. It has been also shown that without using any extra energy sources, switching can be done by changing the displacement of the spring. The same concept is applied to change the assistive force during passive rehabilitation, thus reducing the energy consumtion during this mode. All these features make the hardware structure much more attractive than existing exoskeletons. We aim to miniaturize the exoskeleton with multi degree-of-freedom for upper limb rehabilitation.

\section{REFERENCES}

[1] "State of the Nation: stroke statistics", Stroke Association, 2017. [Online]. Available: https://www.stroke.org.uk/resources/state-nationstroke-statistics. [Accessed: Jan- 2017].

[2] W. Chang and Y. Kim, "Robot-assisted Therapy in Stroke Rehabilitation", Journal of Stroke, vol. 15, no. 3, pp. 174-181, 2013.

[3] N. Takeuchi and S. Izumi, "Rehabilitation with Poststroke Motor Recovery: A Review with a Focus on Neural Plasticity", Stroke Research and Treatment, vol. 2013, pp. 1-13, 2013.

[4] E.T. Wolbrecht, V. Chan, D.J. Reinkensmeyer, and J.E. Bobrow, "Optimizing compliant, model-based robotic assistance to promote neurorehabilitation", IEEE Transactions on Neural Systems and Rehabilitation Engineering, vol. 16, no. 3, pp.286-297, June 2008.

[5] S. Manna and V. Dubey, "Upper Arm Exoskeletons-What specifications will meet users' acceptability?", in Robotics: New Research, 1st ed., D. Fisher, Ed. New York: Nova Science Publisher, 2017, pp. 123-169.

[6] N. Nazmi, M.A.A. Rahman, S.I. Yamamoto, S.A. Ahmad, H. Zamzuri, and S.A. Mazlan, "A review of classification techniques of EMG signals during isotonic and isometric contractions", Sensors, vol. 16, no. 8, pp. 1-28, August 2016.

[7] E. Koyas, E. Hocaoglu, V. Patoglu, and M. Cetin, "Detection of intention level in response to task difficulty from EEG signals" in IEEE International Workshop on Machine Learning for Signal Processing (MLSP), Southampton, UK, 2013, pp. 1-6.

[8] L. Marchal-Crespo, and D. J. Reinkensmeyer, "Review of control strategies for robotic movement training after neurologic injury", Journal of neuroengineering and rehabilitation, vol. 6, no. 1, pp. 1-15, June 2009.

[9] E. T. Wolbrecht, V. Chan, V. Le, S. C. Cramer, D. J. Reinkensmeyer, J. E. Bobrow, "Real-time computer modeling of weakness following stroke optimizes robotic assistance for movement therapy", in 3rd International IEEE/EMBS Conference on Neural Engineering, HI, USA, 2007, pp. 152-158.

[10] A. Handley, P. Medcalf, K. Hellier, and D. Dutta, "Movement disorders after stroke", Age and ageing, vol. 38, no. 3, pp.260-266, March 2009.

[11] Z. Luo, G. Wu, X. Li. and J. Shang, "August. Control Strategy and Experiment of a Novel Hydraulic-Driven Upper Extremity Exoskeleton", in International Conference on Intelligent Robotics and Applications, China, 2017, pp. 51-62.

[12] T. Kosaki, K. Atsuumi, Y. Takahashi, and S. Li, "A pneumatic arm power-assist system prototype with EMG-based muscle activity detection", in IEEE International Conference on Mechatronics and Automation (ICMA), Japan, 2017, pp. 793-798.

[13] V. Dubey and S. Agrawal, "Study of an upper arm exoskeleton for gravity balancing and minimization of transmitted forces", Proceedings of the Institution of Mechanical Engineers, Part H: Journal of Engineering in Medicine, vol. 225, no. 11, pp. 1025-1035, September 2011.

[14] S. Housman and V. Le, "Arm-training with T-WREX after chronic stroke: preliminary results of a randomized controlled trial", in IEEE 10th International Conference on Rehabilitation Robotics, Noordwijk, The Netherlands, 2017, pp. 562-568.

[15] J. Li, R. Zheng, Y. Zhang and J. Yao, "iHandRehab: An interactive hand exoskeleton for active and passive rehabilitation", in 2011 IEEE International Conference on Rehabilitation Robotics, ETH Zurich Science City, Switzerland, 2011, pp. 1-6.

[16] L. Peng, Z. Hou, L. Peng, L. Luo and W. Wang, "Robot assisted rehabilitation of the arm after stroke: prototype design and clinical evaluation”, Science China Information Sciences, vol. 60, no. 7, pp. 1-7, July 2017. 